ISSN : 0852-2626

\title{
JURNAL ZOOTEK
}

Volume 37

Nomor 1 Januari 2017

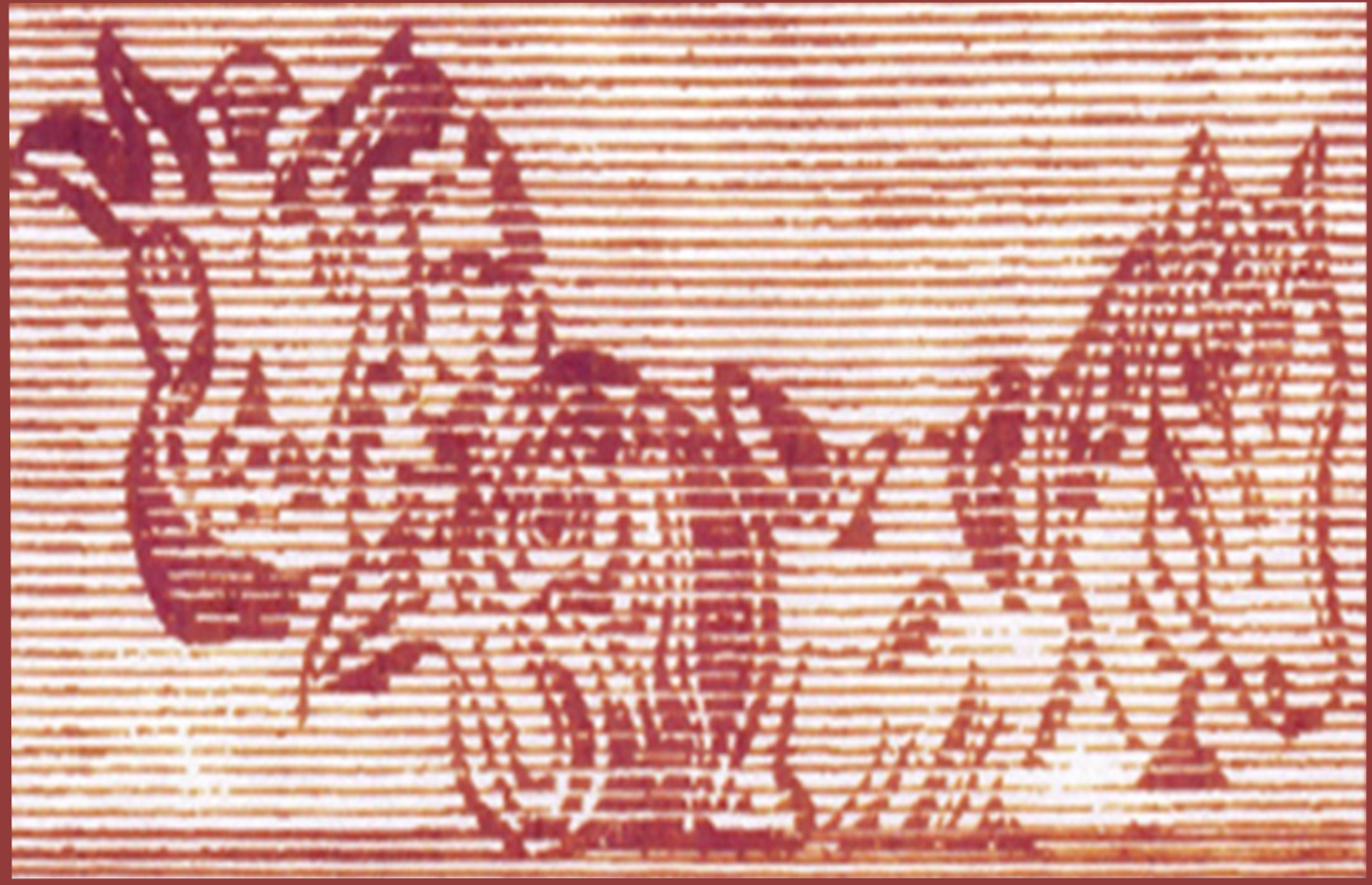

media informasi civitas academica fakultas peternakan universitas sam ratulangi 


\title{
JURNAL ZOOTEK
}

Jurnal Ilmiah Fakultas Peternakan
Universitas Sam Ratulangi Manado

\section{Editor Kepala}

Umar Paputungan

Editor Pelaksana

Erwin Wantasen

Erwin H.B. Sondakh

Malcky M. Telleng

\author{
Editor Ahli \\ Jet S. Mandey (Universitas Sam Ratulangi) \\ Femi H. Elly (Universitas Sam Ratulangi) \\ Rinny J. Leke (Universitas Sam Ratulangi) \\ Meity Sompie (Universitas Sam Ratulangi) \\ Osfar Sofjan (Universitas Brawijaya) \\ Wiludjeng Roessali (Universitas Diponegoro) \\ Joelal Achmadi (Universitas Diponegoro) \\ Yusuf Leonard Henuk (Universitas Sumatera Utara) \\ Amirudin D. Malewa (Universitas Tadulako)
}

\author{
Editor Teknis \\ Jerry A.D. Kalele \\ Mursye N. Regar
}

\section{Penerbit dan Alamat Redaksi}

Jurnal Zootek Fakultas Peternakan UNSRAT, Manado

Jl. Kampus Unsrat, Bahu, Manado, 95115

https://ejournal.unsrat.ac.id/index.php/zootek/issue/archive email: jzootek@yahoo.com 
DAFTAR ISI

KUALITAS KARKAS AYAM PEDAGING YANG DIBERI RANSUM

$1-7$

MENGANDUNG LIMBAH SAWI

Jola J.M.R. Londok, John E.G. Rompis, Claudya Mangelep

PENGGANTIAN SEBAGIAN PAKAN DENGAN TEPUNG LIMBAH SAWI

$8-14$

PUTIH (Brassica pekinensia L) TERHADAP PERFORMANS BROILER

Claudia Mangelep, F. R. Wolayan, M. R. Imbar, I. M. Untu

PENGARUH KONSENTRASI DAN LAMA PERENDAMAN A. pintoi

DENGAN URIN TERNAK SAPI TERHADAP PERTUMBUHAN TANAMAN

A. pintoi

Novalia Lumban Gaol, Ch. L. Kaunang, Rustandi ., F. Dompas

PENGARUH DEFOLIASI DAN LEVEL PUPUK NITROGEN TERHADAP

$25-32$

PERFORMANS RUMPUT Brachiaria humidicola (Rendle) Schweick cv. Tully

Rhama Rizky Ningalo, Rustandi ., David A. Kaligis, Nurhalan Bawole

PERBANDINGAN NILAI BIOLOGIS PAKAN LOKAL DAN IMPOR PADA

$33-40$

ANAK KUDA PACU INDONESIA

Cicilia M Manarisip, Y L.R Tulung, W B Kaunang, $R$ A.V Tuturoong

NILAI RETENSI NITROGEN DAN ENERGI METABOLIS BROILER YANG

DIBERI RANSUM TEPUNG LIMBAH SAWI PUTIH (Brassica rapa L. subsp.

pekinensis)

Fransisca The, J S Mandey, Y H.S Kowel, M N Regar

PERTAMBAHAN BERAT BADAN, JUMLAH KONSUMSI DAN EFISIENSI

PENGGUNAAN PAKAN BABI FASE GROWER SAMPAI FINISHER YANG

$50-61$

DIBERI GULA AREN (Arenga pinnata Merr) DALAM AIR MINUM

Wenny R Poluan, Petrus R.R.I Montong, Jantje F Paath, Vonny R.W Rawung

PERFORMANS BURUNG PUYUH (Coturnix - coturnix japonica) YANG

DIBERIKAN TEPUNG KEONG SAWAH (Pila ampullacea) SEBAGAI

PENGGANTI TEPUNG IKAN DALAM RANSUM

Fransela The, Ch. L. K. Sarajar, M. E. R. Montong, M. Najoan

ANALISIS MARGIN PEMASARAN AYAM BROILER DI PASAR

TRADISIONAL KOTA MANADO

Yudianto Mandak, B. Rorimpandey, P O.V Waleleng, F N.S Oroh

PENGARUH PEMANFAATAN BOKASHI FESES SAPI TERHADAP

$80-87$

PERTUMBUHAN SORGUM VARIETAS KAWALI

Susisusanty Imban, A Rumambi, S S Malalantang

PENGARUH PEMANFAATAN PUPUK BOKASI FESES SAPI TERHADAP

$88-95$

PRODUKSI SORGUM VARIETAS KAWALI

Edward Tacoh, A. Rumambi, W. B. Kaunang

PENGARUH BOBOT TELUR TETAS ITIK TERHADAP PERKEMBANGAN

EMBRIO, FERTILITAS DAN BOBOT TETAS

Sarini Paputungan, Lucia J. Lambey, Linda S. Tangkau, Jaqualine Laihad 
PENGARUH TINGGI DAN JARAK WAKTU PEMOTONGAN RUMPUT

$117-124$

GAJAH DWARF (pannisetum purpureum cv. Mott) TERHADAP

PERTUMBUHAN VEGETATIF DAN PRODUKSI BAHAN KERING

Santia ., Selvie D. Anis, Charles L Kaunang

PENAMPILAN PRODUKSI AYAM RAS PETELUR MB 402 YANG DIBERI

RANSUM MENGANDUNG MINYAK LIMBAH IKAN CAKALANG

(Katsuwonus pelamis L)

Karlia S. Walukow, J. Laihad, Jein Rinny Leke, M. Montong

PERTUMBUHAN VEGETATIF BROWN MIDRIB (BMR) SORGUM PADA

TINGKAT NAUNGAN BERBEDA DAN KEPADATAN POPULASI

Yohanes Barry Kaligis, Ch. L Kaunang, D A Kaligis, Rustandi - -

PENGARUH PENGGUNAAN MOLASES SEBAGAI SUMBER ENERGI

PAKAN PENGUAT DALAM RANSUM TERHADAP PERTUMBUHAN

TERNAK KELINCI

Sumarni Wuysang, C. A. Rahasia, J. F. Umboh, Y. L. R. Tulung

PENGARUH PENAMBAHAN MOLASES TERHADAP KUALITAS FISIK

DAN KIMIA SILASE KULIT PISANG SEPATU (Mussa paradisiaca

formatypica)

Arlen Larangahen, B. Bagau, M. R. Imbar, H. Liwe

EKSPLORASI FUNGI MIKORIZA ARBUSKULA (FMA) PADA RIZOSFIR HIJAUAN PAKAN

Rifa E. Ansiga, A. Rumambi, D. A. Kaligis, I. Mansur, W. Kaunang

PENGARUH NAUNGAN DAN PEMUPUKAN NITROGEN TERHADAP

$125-135$

KARAKTERISTIK MORFOLOGIS RUMPUT GAJAH DWARF (Pennisetum purpureum cv Mott)

Charel Rily Rellam, S. Anis, A. Rumambi, Rustandi .

PENAMPILAN TERNAK KUDA BENDI DI KECAMATAN TOMPASO

KABUPATEN MINAHASA

Santie H. Turangan 